\title{
A competitive comparison of multiparameter stacking
}

\section{operators}

\author{
J. Walda*, B. Schwarz ${ }^{\dagger}$ and D. Gajewski* \\ * University of Hamburg, Bundesstrasse 55, D-20146 Hamburg, Germany. ${ }^{\dagger}$ University of \\ Oxford, South Parks Road, Oxford OX1 3AN, United Kingdom. E-mail: \\ jan.walda@uni-hamburg.de; benjamin.schwarz@earth.ox.ac.uk; \\ dirk.gajewski@uni-hamburg.de.
}

(April 19, 2017)

Running head: Comparison of stacking operators

\begin{abstract}
The classical common-midpoint stack, which sums along offsets, suffers in challenging environments where the acquisition is sparse. In the past, several multiparameter stacking techniques were introduced that incorporate many neighboring common-midpoints during summation. This increases data redundancy and reduces noise. Multiparameter methods that can be parameterized by the same wavefront attributes are multifocusing, the commonreflection-surface (CRS), implicit CRS and non-hyperbolic CRS. CRS-type operators use a velocity shift mechanism to account for heterogeneity by changing the slope of the asymptote. Multifocusing on the other hand uses a different mechanism: a shift of reference time while preserving the slope of the asymptote. We formulate multifocusing such that it uses the same mechanism as the CRS-type operators and compare them on a marine data set. In turn, we investigate the behavior of time-shifted versions of the CRS-type approximations. In order to provide a fair comparison, we use a global optimization technique, differential
\end{abstract}


evolution, which allows to accurately estimate a solution without an initial guess solution. Our results show, that the velocity shift mechanism performs, in general, better than the one incorporating a time shift. The double-square-root operators are also less sensitive to the choice of aperture. They perform better in the case of diffractions than conventional hyperbolic CRS, and this fact is in good agreement with previous works. Since in our study, the non-hyperbolic CRS is of almost the same computational cost as the one of conventional hyperbolic CRS but generally leads to a superior fit, we recommend its use in future. 


\section{INTRODUCTION}

The common-midpoint (CMP) stack by Mayne (1962) has been successfully used for half a century and is still a common strategy. This method is very robust in most cases but does not perform well in very noisy data. Alternative methods which use more traces to utilize data redundancy and reduce noise have been proposed over the last two decades. Some of those can be parameterized with the same attributes. In this work we focus on four multiparameter stacking approaches: multifocusing (MF, Gelchinsky et al., 1999; Landa et al., 2010), the common-reflection-surface (CRS) stack (Jäger et al., 2001), nonhyperbolic CRS (nCRS, Fomel and Kazinnik, 2013) and implicit CRS (iCRS, Schwarz et al., 2014). These stacking approaches can all be expressed in terms of three wavefront attributes (Hubral, 1983) but differ in their mathematical structure. Comparisons between these methods have been done in the past (e.g., Dell et al., 2013; Schwarz and Gajewski, 2017). However, some promising stacking methods were not included in these previous studies. Dell et al. (2013) compared the CRS variants using a normal-moveout (NMO) velocity guide function to estimate the attributes. In their study conflicting dips, which often are mainly caused by diffractions, were not carefully accounted for. It was shown that double-square-root operators perform better for diffraction events by Schwarz et al. (2015). Due to their comparably low amplitudes diffractions are often masked by stronger primary reflections. The full potential of higher-order CRS-type approximations can therefore not be revealed, when conflicting dips are not properly treated. In addition, although a quantitative comparability is generally difficult to achieve, a comparison of the computational efficiency, thus far, has not been addressed in this context.

Following the work of De Bazelaire (1988), Schwarz and Gajewski (2017) introduced 
a new parameterization to account for heterogeneity for CRS-type stacking operators in terms of time and velocity shifts. Multifocusing, in contrast to all other approaches, utilizes a different mechanism, a time shift, to perturb the moveout, whereas the other methods shift the velocity in order to account for overburden heterogeneity. Schwarz and Gajewski (2017) also introduced a recipe to translate time shifts into velocity shifts and vice versa, which allows the comparison of all operators in the same domain. However, in this study the authors used a synthetic model and estimated attributes based on an initial solution obtained by the so called pragmatic approach (Jäger et al., 2001) which can be inaccurate for complex geology (Walda and Gajewski, 2015).

Since in all previous studies, hyperbolic and higher-order CRS expressions including multifocusing were formulated in terms of the two aforementioned mechanisms, they were not compared on common ground, i.e., the gained results are hard to appreciate. In this work, we present an unbiased comparison of the double-square-root operators and conventional hyperbolic CRS for both possible mechanisms consistently, using a global optimization scheme and a sophisticated conflicting dip processing (Walda and Gajewski, 2015) on an industrial field data set compromising many diffracted events.

\section{MULTIPARAMETER STACKING}

The CMP method (Mayne, 1962) stacks data along a traveltime curve in offset direction

$$
S\left(t_{0}, m_{0}\right)=\int P\left(t\left(t_{0}, h\right), m_{0}, h\right) \mathrm{d} h \quad .
$$

The stack is denoted by $S ; P$ is the prestack data; $t_{0}$ is the zero-offset traveltime; $h$ the halfoffset and $m_{0}$ the zero-offset coordinate. This method uses only data redundancy in offset direction which becomes problematic if only few traces are available. Challenging data, 
where CMP processing does not produce desired results, benefit from stacking techniques where the summation is carried out in both offset and midpoint direction

$$
S\left(t_{0}, m_{0}\right)=\iint P\left(t\left(t_{0}, \Delta m, h\right), m, h\right) \mathrm{d} m \mathrm{~d} h .
$$

In Equation 2, the quantity $\Delta m=m_{0}-m$ is the midpoint displacement. Since neighboring midpoints $m$ are stacked into the midpoint $m_{0}$ as well, a higher amount of traces are summed which results in a better signal-to-noise ratio. However, the traveltime description depends on more parameters compared to classical CMP stacking. Hubral (1983) introduced wavefront attributes (Figure 1), which can be used as a parameterization to describe the required traveltime surface $t\left(t_{0}, \Delta m, h\right)$. Traveltime operators that use these attributes can be classified as common-reflection-surface (CRS) type operators. They differ in their mathematical structure which stem from underlying assumptions and deviations. While CRS itself is a hyperbolic single-square-root traveltime expression, the other three operators discussed in the following sections are double-square-root operators which better describe scattering of highly curved structures like diffractions.

\section{Multifocusing}

Planar multifocusing introduced by Gelchinsky et al. (1999) and revisited by Landa et al. (2010) attempts to express the traveltime in terms of the traveltime of a central ray and two corrections at the source and receiver position from a paraxial ray. To achieve this, a parameter to focus the NIP and $N$ wave for a planar reflector is required which reads

$$
\begin{aligned}
R^{ \pm} & =\frac{1 \pm \gamma}{\frac{1}{R_{N}} \pm \frac{\gamma}{R_{N I P}}}, \\
\gamma & =\frac{\Delta x^{+}-\Delta x^{-}}{\Delta x^{+}+\Delta x^{-}+2 \frac{\sin \alpha}{R_{N I P}} \Delta x^{+} \Delta x^{-}},
\end{aligned}
$$


where $\Delta x^{+}=\Delta m-h, \Delta x^{-}=\Delta m+h$. The quantity $v_{0}$ is the near surface velocity and $\gamma$ is called focusing parameter, which together with the equation

$$
\begin{aligned}
t\left(t_{0}, \Delta m, h\right)=t_{0} & +\frac{\sqrt{\left(R^{+}\right)^{2}+2 R^{+} \Delta x^{+} \sin \alpha+\left(\Delta x^{+}\right)^{2}}-R^{+}}{v_{0}} \\
& +\frac{\sqrt{\left(R^{-}\right)^{2}+2 R^{-} \Delta x^{-} \sin \alpha+\left(\Delta x^{-}\right)^{2}}-R^{-}}{v_{0}},
\end{aligned}
$$

leads to the multifocusing operator. However, multifocusing as presented here and in literature uses a time shift to account for heterogeneity. Therefore, previous comparisons of multifocusing with other CRS type operators as in (Tygel et al., 1999; Landa et al., 2010; Fomel and Kazinnik, 2013) are not on a common ground.

\section{Common-reflection-surface}

The CRS stack is a multiparameter stacking technique (Müller, 1999; Mann et al., 1999; Jäger et al., 2001; Mann, 2002) that describes an event in the vicinity of the zero-offset sample by a second-order traveltime approximation (Figure 2).

The CRS operator is formulated in terms of three wavefront attributes, which are related to two hypothetical one-way experiments as illustrated in Figure 1. The resulting two waves are described by the angle of emergence $\alpha$ of the zero-offset ray and the corresponding radii of curvature: $R_{N}$ for the normal (N) wave and $R_{N I P}$ for the normal-incidence-point (NIP) wave (Hubral, 1983). The $\mathrm{N}$ wave is generated by a fictitious exploding reflector experiment around the normal-incidence-point. The NIP wave is generated by a fictitious point source placed at the normal-incidence-point.

Using the notation by Fomel and Kazinnik (2013), the CRS formula in its hyperbolic 
expression can be written as

$$
\begin{aligned}
t\left(t_{0}, \Delta m, h\right) & =\sqrt{F\left(\Delta m, t_{0}\right)+b_{2} h^{2}} \\
F\left(t_{0}, \Delta m\right) & =\left(t_{0}+a_{1} \Delta m\right)^{2}+a_{2} \Delta m^{2}
\end{aligned}
$$

The coefficients $a_{1}, a_{2}$ and $b_{2}$ are related to the wavefront attributes $\alpha, R_{N I P}$ and $R_{N}$ since

$$
\begin{aligned}
& a_{1}=\frac{2 \sin \alpha}{v_{0}} \\
& a_{2}=\frac{2 t_{0} \cos ^{2} \alpha}{v_{0} R_{N}} \\
& b_{2}=\frac{2 t_{0} \cos ^{2} \alpha}{v_{0} R_{N I P}},
\end{aligned}
$$

where $v_{0}$ is the near surface velocity. This notation is different from the notation usually used in CRS literature but allows us a good link to the nCRS operator considered in the next section.

\section{Non-hyperbolic common-reflection-surface}

Fomel and Kazinnik (2013) introduced another extension of the conventional CRS approach. In contrast to iCRS (see below) the non-hyperbolic common-reflection-surface (nCRS) method assumes a hyperbolic reflector and, for a homogeneous background, provides the exact traveltimes.

The nCRS operators reads

$$
t\left(t_{0}, \Delta m, h\right)=\sqrt{\frac{F\left(t_{0}, \Delta m\right)+c h^{2}+\sqrt{F\left(t_{0}, \Delta m-h\right) F\left(t_{0}, \Delta m+h\right)}}{2}}
$$

where $F$ is defined according to Equation 7 and $c=2 b_{2}+a_{1}^{2}-a_{2}$ accounts for the asymmetry of the source-receiver traveltime contributions. From an implementation point of view, it requires very few additional computations compared to CRS (see Equation 6) which makes it very easy and efficient to implement in existing CRS codes. 


\section{Implicit common-reflection-surface}

The implicit common-reflection-surface (iCRS) stack is a further development of the commonreflection-surface (CRS) stack. In contrast to the conventional CRS technique, iCRS, similar to Höcht et al. (1999), and Landa et al. (2010), assumes a circular reflector in the subsurface.

The iCRS operator is another multiparameter stacking technique which was derived by Schwarz et al. (2014). It assumes a locally circular reflector as shown in Figure 3. It depends on three parameters of the circle $\left(x_{c}, H\right.$ and $\left.R\right)$ as well as the background velocity of the medium $V$ and reads

$$
\begin{aligned}
t_{s}(\Delta m, h) & =\frac{1}{V} \sqrt{\left(\Delta m-h-\Delta x_{c}-R \sin \theta\right)^{2}+(H-R \cos \theta)^{2}} \\
t_{g}(\Delta m, h) & =\frac{1}{V} \sqrt{\left(\Delta m+h-\Delta x_{c}-R \sin \theta\right)^{2}+(H-R \cos \theta)^{2}} \\
t(\Delta m, h) & =t_{s}(\Delta m, h)+t_{g}(\Delta m, h),
\end{aligned}
$$

where $t_{s}$ is the traveltime from the source to the reflection point, $t_{g}$ from the reflection point to the receiver, $\Delta x_{c}$ is the displacement of the circle relative to the central midpoint location $m_{0}$ and $\theta$ the reflection point angle on the circle. This angle has to be computed additionally. It depends on the traveltimes $t_{s}$ and $t_{g}$

$$
\tan \theta=\tan \theta_{0}+\frac{h}{H} \frac{t_{s}-t_{g}}{t_{s}+t_{g}}
$$

and can be solved in an iterative fashion with a first guess provided by the zero-offset ray. In this case, the dependence on $t_{s}$ and $t_{g}$ vanishes

$$
\tan \theta_{0}=\frac{\Delta m-\Delta x_{c}}{H} .
$$

Schwarz et al. (2014) showed that a single iteration is usually already sufficient to achieve an accurate fit in heterogeneous media. The parameters of the circle can be related to the 
CRS wavefront attributes by

$$
\begin{aligned}
V_{N M O} & =\sqrt{\frac{2 v_{0} R_{N I P}}{t_{0} \cos ^{2} \alpha}} \\
V & =\frac{V_{N M O}}{\sqrt{1+\frac{V_{N M O}^{2}}{v_{0}^{2}} \sin ^{2} \alpha}} \\
\Delta x_{c} & =\frac{-R_{N I P} \sin \alpha}{\cos ^{2} \alpha\left(1+\frac{V_{N M O}^{2}}{v_{0}^{2}} \sin ^{2} \alpha\right)} \\
H & =\frac{v_{0} R_{N I P}}{V_{N M O} \cos ^{2} \alpha\left(1+\frac{V_{N M O}^{2}}{v_{0}^{2}} \sin ^{2} \alpha\right)} \\
R & =\frac{\frac{v_{0} R_{N}}{V_{N M O} \cos ^{2} \alpha}-\frac{V_{N M O} t_{0}}{2}}{\sqrt{1+\frac{V_{N M O}^{2}}{v_{0}^{2}} \sin ^{2} \alpha}} . \\
&
\end{aligned}
$$

Therefore, the iCRS traveltime surface can be written in terms of the CRS wavefront

attributes $\alpha, R_{N I P}$ and $R_{N}$. Please note that similar to conventional CRS and nCRS the iCRS moveout, i.e. $\Delta t=t-t_{0}$, depends on the reference traveltime $t_{0}$. For multifocusing, in contrast, $\Delta t$ is not a function of $t_{0}$. This is a consequence of the different ways to account for heterogeneity, i.e., time shift or velocity shift.

\section{TIME SHIFT AND VELOCITY SHIFT}

All presented approximations describe paraxial traveltimes and are functions of the same wavefront attributes

$$
t=t\left(t_{0}, \alpha, R_{N I P}, R_{N}\right)
$$

In the recent work of Schwarz and Gajewski (2017), the authors showed that the multifocusing moveout, despite being parameterized in terms of the same kinematic wavefront attributes, behaves differently from all other approaches when heterogeneity is present. In this study Schwarz and Gajewski (2017) found, that a time shift is responsible for perturbing the moveout, whereas for the moveout of CRS-type operators, a velocity shift accounts 
for velocity changes in the overburden. In Figure 4 the conceptual difference between either shifting the time or the velocity is illustrated. Schwarz and Gajewski (2017) introduced a simple recipe to transform time shifts to velocity shifts and vice versa,

$$
\frac{1}{v_{\text {shift }}^{2}}=\left(\frac{\sin \alpha}{v_{0}}\right)^{2}+\frac{t_{0}}{t_{\text {shift }}}\left[\frac{1}{v_{0}^{2}}-\left(\frac{\sin \alpha}{v_{0}}\right)^{2}\right],
$$

where $t_{0}$ is the zero-offset reference traveltime and $t_{\text {shift }}=2 R_{N I P} / v_{0}$ the shifted zero-offset traveltime. Equation 15 connects both parameterizations. The time shift mechanism can be illustrated in the framework of geometrical optics and since the dip is naturally accounted for, Equation 15 can be considered as a generalized osculating equation (compare De Bazelaire, 1988). This allows to freely choose the mechanism to account for heterogeneity. However, this also means each operator introduced in literature has two versions. In literature four CRS type operators are available, namely multifocusing (MF), CRS, nonhyperbolic CRS (nCRS) and implicit CRS (iCRS). This means there is a choice of eight CRS type traveltime expressions that can be used in Equation 2. In order to evaluate differences in the practical application we apply all operators under the same conditions to marine industrial field data. We use differential evolution (Storn and Price, 1997) as a global optimization technique to estimate the CRS wavefront attributes at every sample with the same aperture for all approximations. Since the velocity at each time sample along a wavelet can change, the velocity-shifted approximations do not suffer from the typical moveout stretch. Furthermore, in contrast to Dell et al. (2013) we do not use a velocity guide function to better evaluate the performance differences of the operators. A guide function would introduce a favorable or unfavorable bias, since velocities might differ, depending on the operator. Since the strongest differences are expected for diffractions, we also account for conflicting dips by dividing the $\alpha$ search space into small clusters. This allows to recognize different events in separate $\alpha$ clusters (Walda and Gajewski, 2015). In the next section we present 
examples of this comparison.

\section{MARINE DATA EXAMPLE}

In order to find the most suitable operator, especially for diffraction imaging, their performances under the very same conditions, as described above, are compared. The sole difference is the operator used for traveltime fitting to calculate the semblance, which is used as the coherence measure in this study.

As a method to gain more insight into the behavior of the operators we consider here the objective function for a specific test case. For this purpose, we consider a test sample indicated by the arrow in Figure 5 . In Figure 6 the $2 \mathrm{D} \alpha-R_{N I P}$ planes of the 3D parameter cube for a constant $R_{N}=1720.73 \mathrm{~m}$ of every operator are plotted. The chosen $R_{N}$ corresponds to the best found value for the event at $22^{\circ}$. The midpoint aperture is $800 \mathrm{~m}$ and the offset-target ratio is approximately $2: 1$. In order to emphasize the diffractions, the semblance is clipped in this plot, since the reflections take considerably higher values than the diffractions. Since this layout is far beyond the hyperbolic limit, the CRS operator cannot fit the diffractions anymore and they are hardly recognizable. Furthermore, the estimated attributes differ as well as the location and amount of maxima in the objective function. This is not so much the case for the higher-order operators, i.e., multifocusing, nCRS and iCRS. They do not differ at the maximum, but only in the noisy part of the objective function where no maximum is present.

To evaluate the presented stacking operators, we use data obtained in the Mediterranean Sea. The data is of good quality (see Figure 7 for an example CMP gather) and, therefore, is suitable for comparisons. 
Since every operator can be formulated in its velocity and time-shifted version, it is necessary to investigate which representation is better suited for stacking. Figure 8 shows the semblance of velocity-shifted nCRS as a reference and the difference plots of the two representations for three exemplarily chosen operators. In the difference plots, a red color represents higher semblance values for the velocity-shifted version whereas blue colors show higher semblance for the time-shifted operator. Independent of the actual traveltime expression, the velocity-shifted versions show a higher coherence almost everywhere and a better fit for the same events.

Figure 9 shows the semblance obtained by nCRS and the respective difference plots of nCRS and the other CRS operators in their velocity-shifted version. Red colors represent a better fit for nCRS, blue colors for CRS, iCRS or multifocusing. The difference plots of nCRS minus iCRS and nCRS minus multifocusing reveal no significant differences. In terms of accuracy they perform similarly on the marine field data. However, they show differences compared to the hyperbolic CRS represented by the difference plot of nCRS minus CRS. Differences for reflections are not visible, which is not surprising, because all considered expressions coincide up to second order. In case of diffractions, mainly red colors appear which means a better fit for nCRS. This is no surprise since diffractions are described more accurately by double-square-root operators. Since the differences of nCRS to iCRS and multifocusing are almost nonexistent, the deviations are the same for all non-hyperbolic operators compared to hyperbolic CRS. The fit of diffractions is better for non-hyperbolic operators. This also shows in the estimated attributes, exemplarily shown for the moveout velocity in Figure 10. A reference velocity field, estimated using nCRS and difference plots of nCRS minus CRS, iCRS and multifocusing are shown. Red colors show a higher moveout velocity of nCRS while blue colors indicate higher moveout velocities for the other 
operators. The biggest differences are visible for nCRS minus CRS. In case of diffractions, CRS shows higher moveout velocities close to the apex while at the diffraction tail, the approximation of nCRS leads to higher moveout velocities, which is more accurate because the semblance of nCRS is higher. nCRS and multifocusing show very similar moveout velocities apart from areas of very low coherence. Interestingly, even though the differences in the coherence between nCRS and iCRS are almost nonexistent, the moveout velocities show small differences. For dipping events, the moveout velocity of iCRS is higher than for nCRS. Unfortunately it is hard to tell, which is more accurate.

The observation that non-hyperbolic operators perform better in case of diffractions than hyperbolic CRS is further supported by a diffraction separation using CRS attributes (see, e.g., Dell and Gajewski, 2011). The results of this application are shown in Figure 11. An excerpt of the data was considered for diffraction separation using nCRS and CRS. The excerpt area is highlighted by the red box. In case of nCRS, more diffractions are visible and imaged more continuously. The arrows highlight diffractions where this can be observed in particular. This observation is consistent with the recent study of Abakumov (2016) who investigated the accuracy of multiparameter stacking operators for specific cases in 3D. Finally, Figure 12 presents a comparison of the computational costs of the operators. The additional computational overhead of the velocity-shifted nCRS compared to CRS is very small (about $5 \%$ ) while the better accuracy for diffractions and lower sensitivity with respect to the aperture are strong benefits (Dell et al., 2013). For the complex field data example, nCRS provides the best trade-off between accuracy, stability and computational effort. 


\section{DISCUSSION}

We have compared multiparameter stacking operators that can be parameterized by the same wavefront attributes. The major differences in the quality of the fit can almost entirely be attributed to the gross mathematical structure of the operator, i.e. hyperbolic or doublesquare-root shape, which suggests that further research should rather be concerned with the topics of parameterization and implementation than with merely developing additional straight-ray approximations. The results show that there is practically no difference in the actual applications between multifocusing, nCRS and iCRS. This requires to formulate multifocusing such that it uses the same mechanism to account for heterogeneity as CRStype operators. The recipe to transform multifocusing to a velocity-shifted formulation and the CRS variants to a time-shifted version leads to an unification of the CRS-type operator. The differentiation between them is connected to the hyperbolic and doublesquare-root shape, if the operators are expressed for the same parameterization, i.e., time shift or velocity shift. In the presented framework of global optimization and conflictingdip handling, the considered traveltime expressions can be replaced by each other without the need to adjust the implementation, which suggests that they should not be viewed as competing concepts, but as representatives of the same CRS family.

All presented operators were compared for a 2D acquisition in this study, which obviously is a drastic simplification. Because out-of-plane contributions and diffractions can only be fully characterized in 3D, the extension of the approximations for this more general case should be a crucial next step. Since for CRS and nCRS, 3D expressions are already explicitly available in literature, there are arguments to use nCRS in the future. In addition, due to the relatively low degree of mathematical complexity, its application turns out to be 
computationally less demanding compared to the other double-square-root expressions.

\section{CONCLUSIONS}

We investigated currently used multiparameter stacking operators, which can be parameterized by CRS wavefront attributes. Our results show, that in general a non-hyperbolic operator should be used rather than the conventional hyperbolic CRS expression, because the non-hyperbolic variants generally provide a slightly better fit and are less dependent on the choice of the search aperture. The question of which specific operator should be used in terms of accuracy cannot be answered conclusively, since multifocusing, iCRS, and nCRS perform similarly well. However, in our global optimization scheme the non-hyperbolic CRS used less computation time, which can be attributed to the fact that its overall mathematical complexity is relatively low compared to the double-square-root alternatives of the implicit CRS and multifocusing operators, which can be formulated in terms of a velocity or time shift. The comparison shows that velocity-shifted versions generally perform better computationally and lead to a more accurate fit. Since multifocusing up to this point appeared in the time shifted version in literature, it is recommended to use it in its velocityshifted version to obtain a better performance in the presence of heterogeneity. However, the velocity-shifted version of nCRS provides the best trade-off between accuracy and com-

putational cost in case of diffractions. Therefore, we suggest to use the nCRS operator to determine wavefront attributes if diffraction imaging is of interest. 


\section{ACKNOWLEDGMENTS}

The work was supported by the sponsors of the Wave Inversion Technology (WIT) consortium and the Federal Ministry for Economic Affairs and Energy of Germany (project number 03SX427B). The marine data was provided by TGS-NOPEC. We would like to thank the Applied Seismics Group Hamburg for continuous discussions and the editors and reviewers for their comments and suggestions. 


\section{REFERENCES}

Abakumov, I., 2016, Systematic analysis of double-square-root-based stacking operators: PhD thesis, Universitt Hamburg, Von-Melle-Park 3, 20146 Hamburg.

De Bazelaire, E., 1988, Normal moveout revisited: Inhomogeneous media and curved interfaces: Geophysics, 53, 143-157.

Dell, S., J. Downes, and T. Hertweck, 2013, Comparison of Non-hyperbolic and Standard CRS Using Complex Field Data: 2013 SEG Annual Meeting, 4357-4361.

Dell, S., and D. Gajewski, 2011, Common-reflection-surface-based workflow for diffraction imaging: Geophysics, 76, S187.

Fomel, S., and R. Kazinnik, 2013, Non-hyperbolic common reflection surface: Geophysical Prospecting, 61, 21-27.

Gelchinsky, B., A. Berkovitch, and S. Keydar, 1999, Multifocusing homeomorphic imaging: Part 1. Basic concepts and formulas: Journal of Applied Geophysics, 42, 229-242.

Höcht, G., E. De Bazelaire, P. Maher, and P. Hubral, 1999, Seismics and optics: hyperbolae and curvatures: Journal of Applied Geophysics, 42, 261281.

Hubral, P., 1983, Computing true amplitude reflections in a laterally inhomogeneous earth: Geophysics, 48, 1051-1062.

Jäger, R., J. Mann, G. Höcht, and P. Hubral, 2001, Common-reflection-surface stack: Image and attributes: Geophysics, 66, 97-109.

Landa, E., S. Keydar, and T. J. Moser, 2010, Multifocusing revisited-inhomogeneous media and curved interfaces: Geophysical Prospecting, 58, 925-938.

Mann, J., 2002, Extensions and Applications of the Common-Reflection-Surface Stack Method: PhD thesis, Logos Verlag, Berlin.

Mann, J., R. Jäger, T. Müller, G. Höcht, and P. Hubral, 1999, Common-reflection-surface 
stack - a real data example: Journal of Applied Geophysics, 42, 301-318.

Mayne, W. H., 1962, Common reflection point horizontal data stacking techniques: Geophysics, 27, 927-938.

Müller, T., 1999, The Common Reflection Surface Stack Method: Seismic Imaging without explicit knowledge of the velocity model: $\mathrm{PhD}$ thesis, University of Karlsruhe.

Schwarz, B., and D. Gajewski, 2017, The two faces of NMO: The Leading Edge, accepted for publication.

Schwarz, B., C. Vanelle, and D. Gajewski, 2015, Shifted Hyperbola Revisited-The Two Faces of NMO: 77th EAGE Conference and Exhibition 2015.

Schwarz, B., C. Vanelle, D. Gajewski, and B. Kashtan, 2014, Curvatures and inhomogeneities: An improved common-reflection approach: Geophysics, 79, S231-S240.

Storn, R., and K. Price, 1997, Differential Evolution - a simple and efficient heuristic for global optimization over continues spaces: Jornal of Global Optimization, 11, 341-359.

Tygel, M., L. T. Santos, and J. Schleicher, 1999, Multifocus moveout revisited: derivations and alternative expressions: Journal of Applied Geophysics, 42, 319-331.

Walda, J., and D. Gajewski, 2015, Common-reflection-surface stack improvement by differential evolution and conflicting dip processing: SEG Technical Program Expanded Abstracts, 3842-3847. 


\section{LIST OF FIGURES}

1 Two hypothetical experiments: The normal-incidence-point wave is shown in the middle with its radius of curvature $R_{N I P}$. The normal wave with the radius of curvature $R_{N}$, is caused by an exploding reflector experiment (right). Both have the angle of emergence $\alpha$ (left).

2 The CRS method stacks along the fat black traveltime surface and assigns the value to the black dot.

3 Geometrical sketch for the derivation of the iCRS operator.

4 Illustration of the two possible mechanisms to perturb the moveout to account for overburden heterogeneity by the CRS operator. The solid lines represent the actual unperturbed (black) and perturbed (red) moveout. For the velocity shift, the slope of the moveouts asymptote (meshed) is changed while the reference time is kept constant. Hence, the heterogeneity is accounted for by a changing asymptote of the traveltime. In case of the time shift, the time of origin of the asymptote is changing while the asymptote of the traveltime surface itself is kept constant. We use a non-vanishing moderate reflector curvature $\left(R_{N I P} / R_{N}=0.5\right)$ and a vanishing reflector inclination at the central reference midpoint.

5 Stacked section in the north east of the profile. The arrow indicates the location of the sample where the objective function is investigated.

6 Objective functions of a) CRS, b) multifocusing, c) nCRS and d) iCRS for the sample shown by an arrow in Figure 5. The offset-target ratio is 2.0 which exceeds the hyperbolic limit significantly. Therefore, the objective function of the conventional CRS operator differs severely from the non-hyperbolic variants. The difference between the individual non-hyperbolic expressions, however, is negligible.

$7 \quad$ A CMP gather from the north east of the profile. 
8 Semblance section obtained by a) the velocity-shifted nCRS operator and difference plots of velocity-shifted operators to their time shifted counterparts for b) CRS, c) multifocusing and d) nCRS. A red color indicates a higher semblance for the velocity-shifted operator, while blue colors indicate a better semblance of time shifted operators.

9 Semblance section obtained by a) the velocity-shifted nCRS operator and difference plots of velocity-shifted nCRS to the other velocity-shifted CRS-type operators: b) CRS, c) multifocusing and d) iCRS. A red color indicates a higher semblance for the velocity-shifted nCRS operator, while blue colors indicate a better semblance for the other velocity-shifted operator.

10 Comparison of the estimated moveout velocities obtained by the different operators. The results from nCRS serve as reference for difference plots, since the differences are small. A red color indicates a higher velocity for the velocity-shifted nCRS operator, while blue colors indicate a lower velocity compared to the other velocity-shifted operator.

11 Stacked section of the fault system. The red box shows the excerpt shown on the right, where a diffraction separation is performed using attributes obtained by the CRS and nCRS operators. Red arrows indicate minor improvements achieved by nCRS compared to CRS. Most improvements concern the diffraction tails which are interesting for diffraction imaging. Multifocusing and iCRS are not shown since they performed similar to nCRS.

12 Comparison of the computation time for each operator in their a) velocity- and b) time-shifted versions. The hyperbolic CRS in the velocity-shifted version is the reference (100 \%). The yellow color represents the calculation time of CRS while the red color is the additional computational cost of the corresponding operator. 

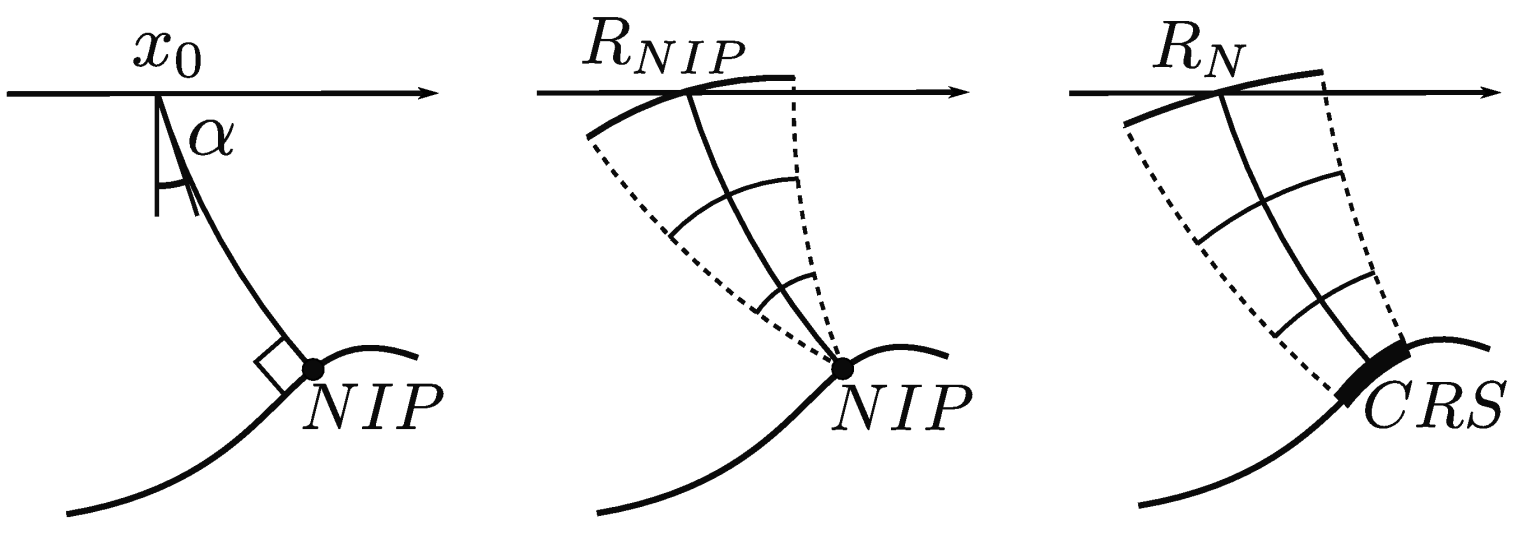

Figure 1: Two hypothetical experiments: The normal-incidence-point wave is shown in the middle with its radius of curvature $R_{N I P}$. The normal wave with the radius of curvature $R_{N}$, is caused by an exploding reflector experiment (right). Both have the angle of emergence $\alpha$ (left).

Walda et al. 


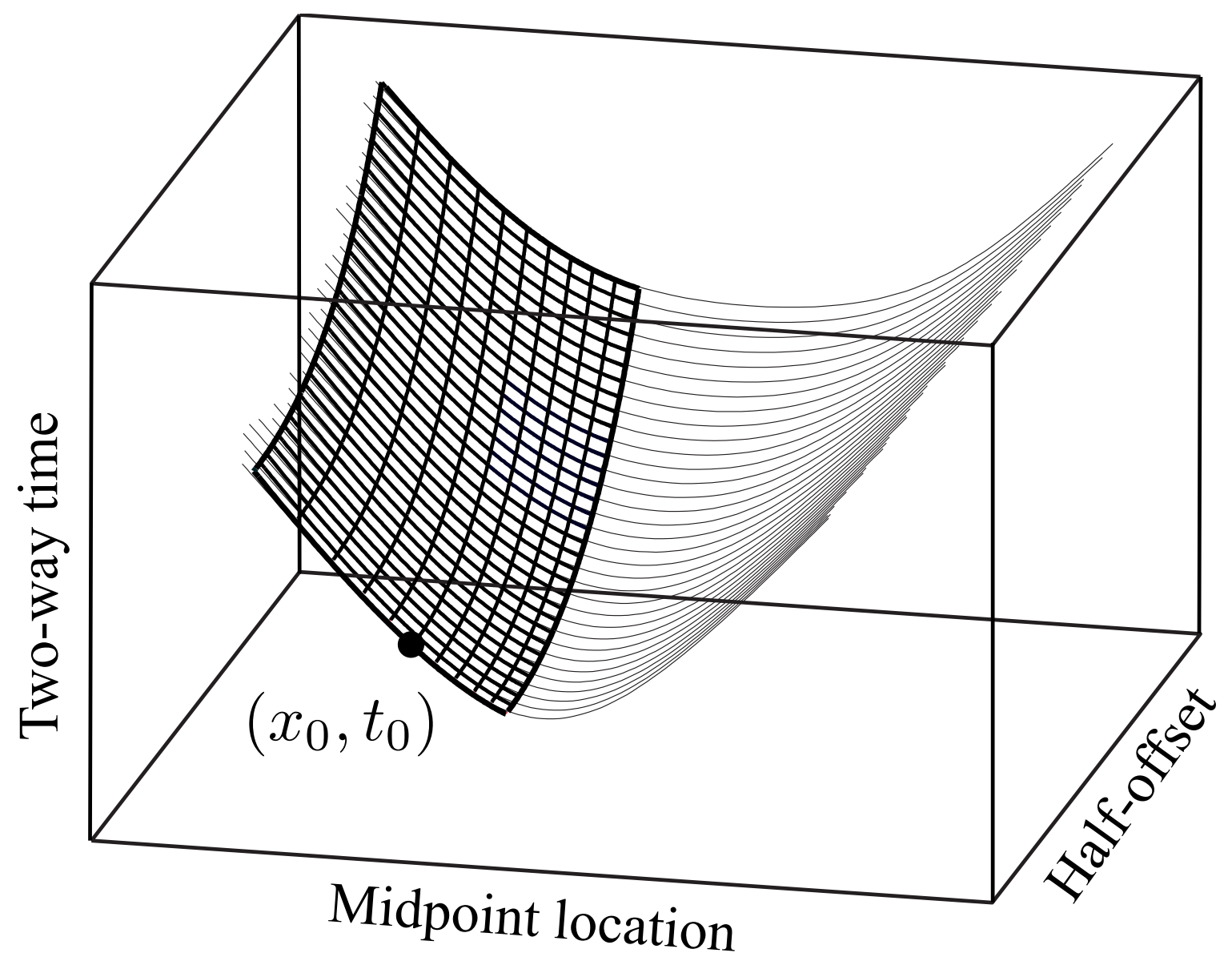

Figure 2: The CRS method stacks along the fat black traveltime surface and assigns the value to the black dot.

Walda et al. - 


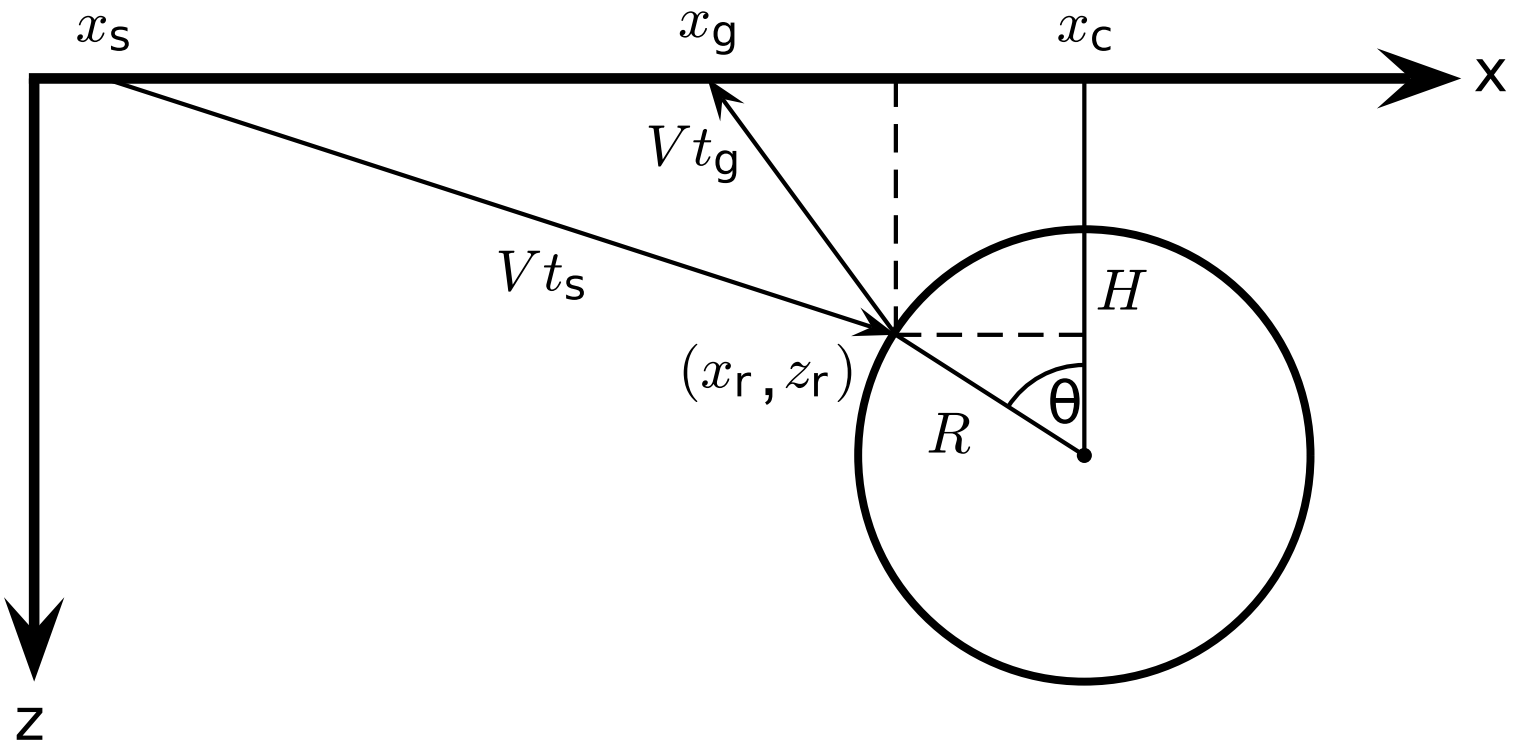

Figure 3: Geometrical sketch for the derivation of the iCRS operator.Walda et al. - 

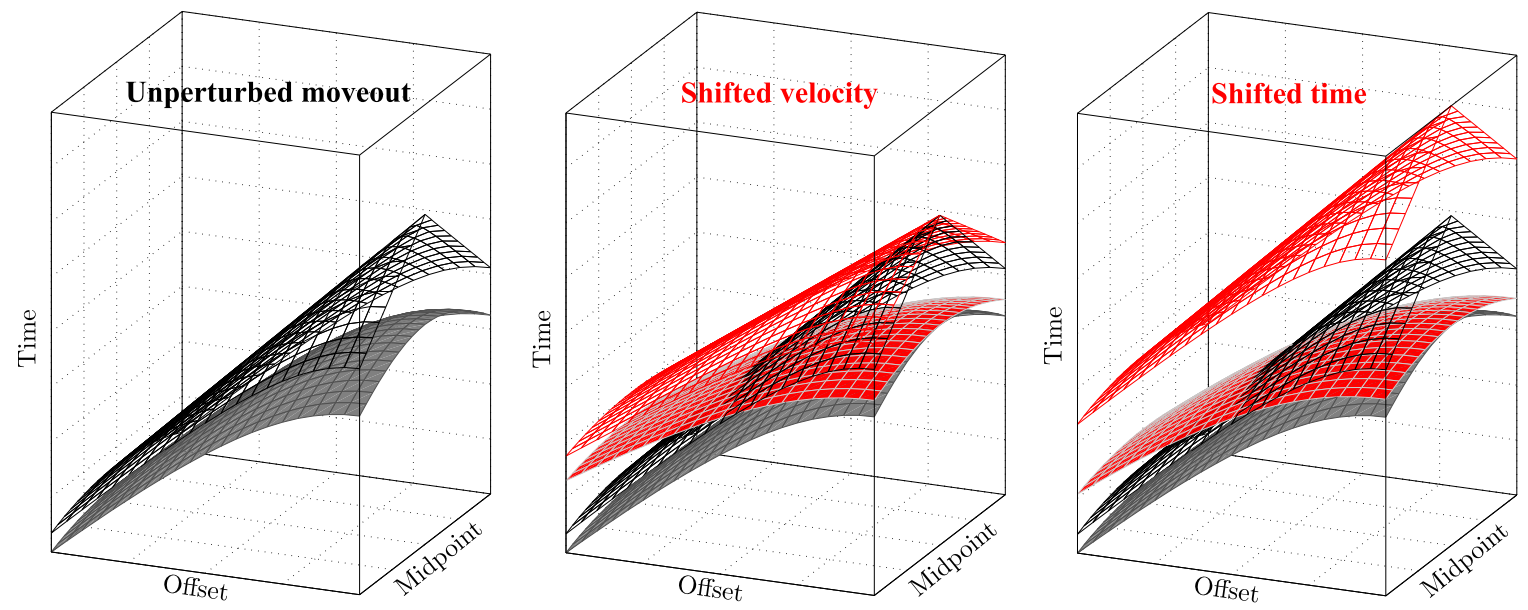

Figure 4: Illustration of the two possible mechanisms to perturb the moveout to account for overburden heterogeneity by the CRS operator. The solid lines represent the actual unperturbed (black) and perturbed (red) moveout. For the velocity shift, the slope of the moveouts asymptote (meshed) is changed while the reference time is kept constant. Hence, the heterogeneity is accounted for by a changing asymptote of the traveltime. In case of the time shift, the time of origin of the asymptote is changing while the asymptote of the traveltime surface itself is kept constant. We use a non-vanishing moderate reflector curvature $\left(R_{N I P} / R_{N}=0.5\right)$ and a vanishing reflector inclination at the central reference midpoint.

Walda et al. 


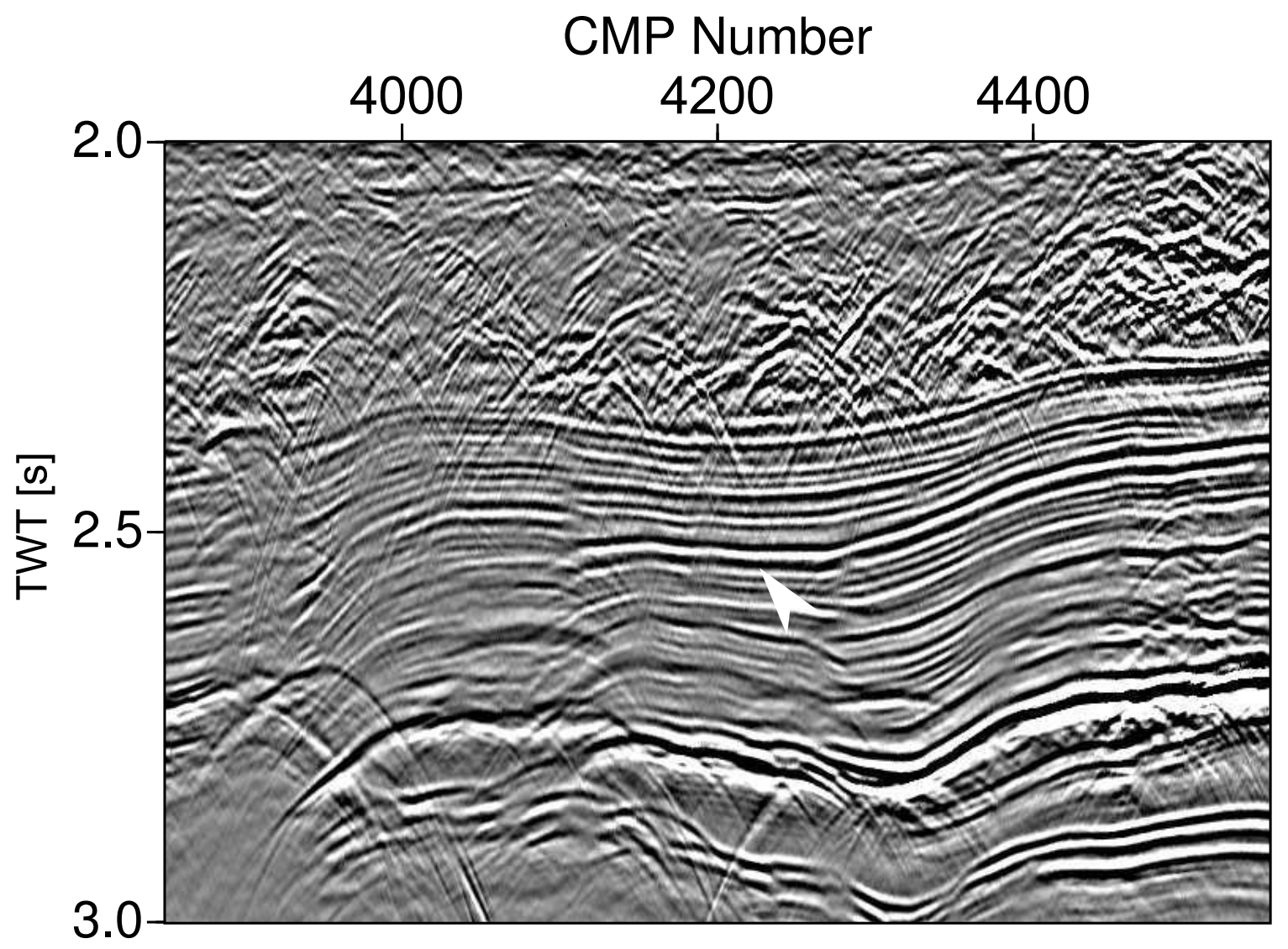

Figure 5: Stacked section in the north east of the profile. The arrow indicates the location of the sample where the objective function is investigated.

Walda et al. 

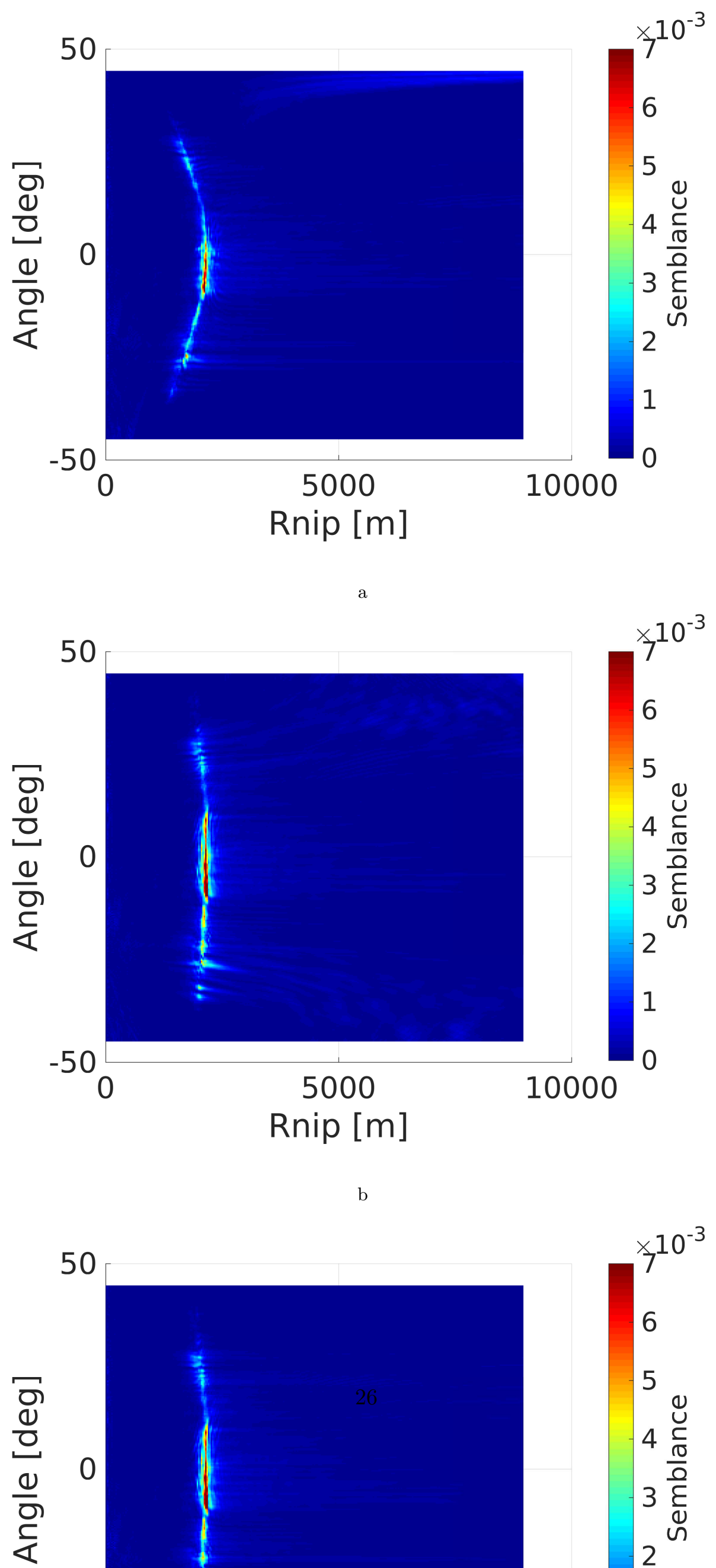


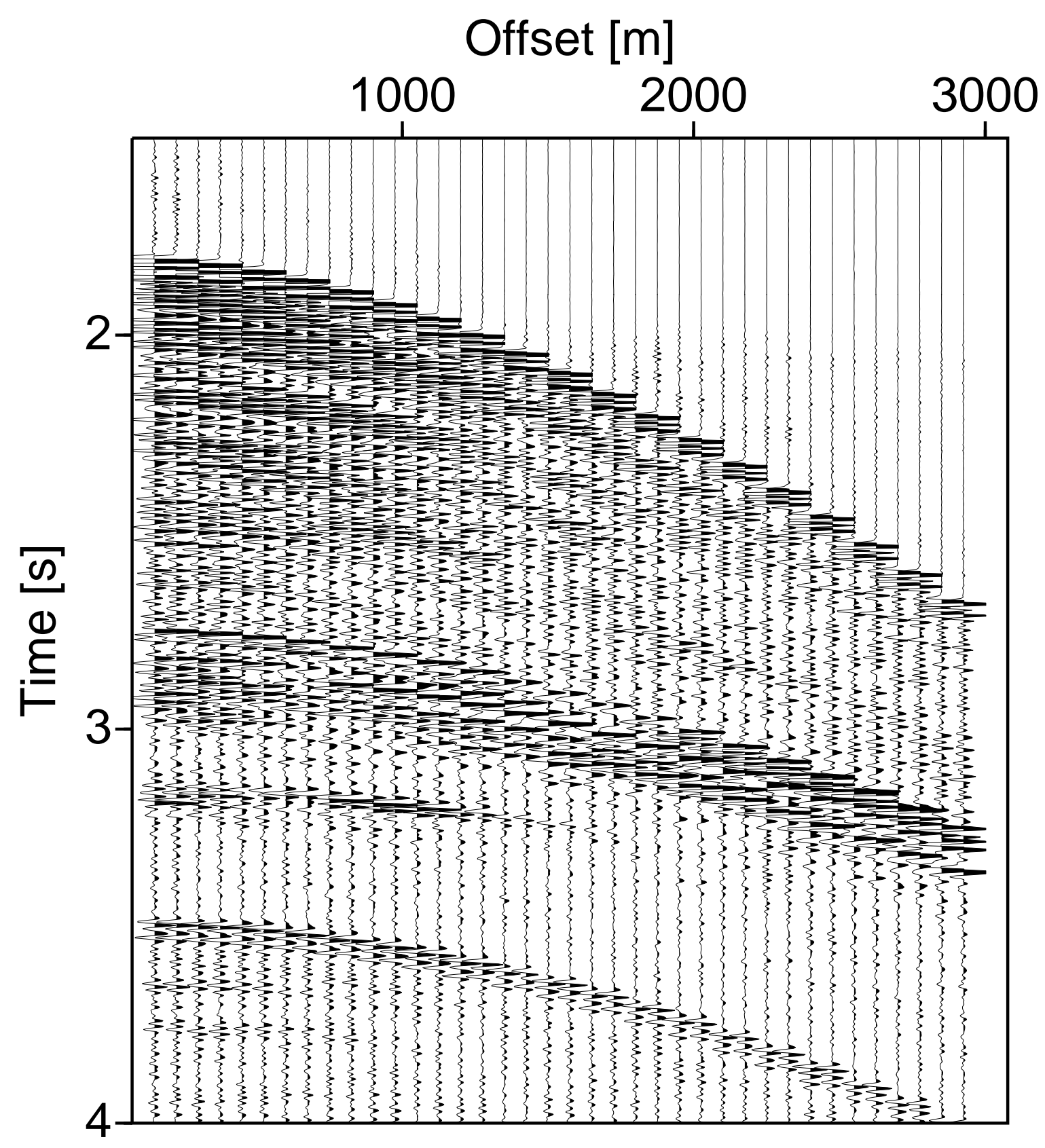

Figure 7: A CMP gather from the north east of the profile.Walda et al. 

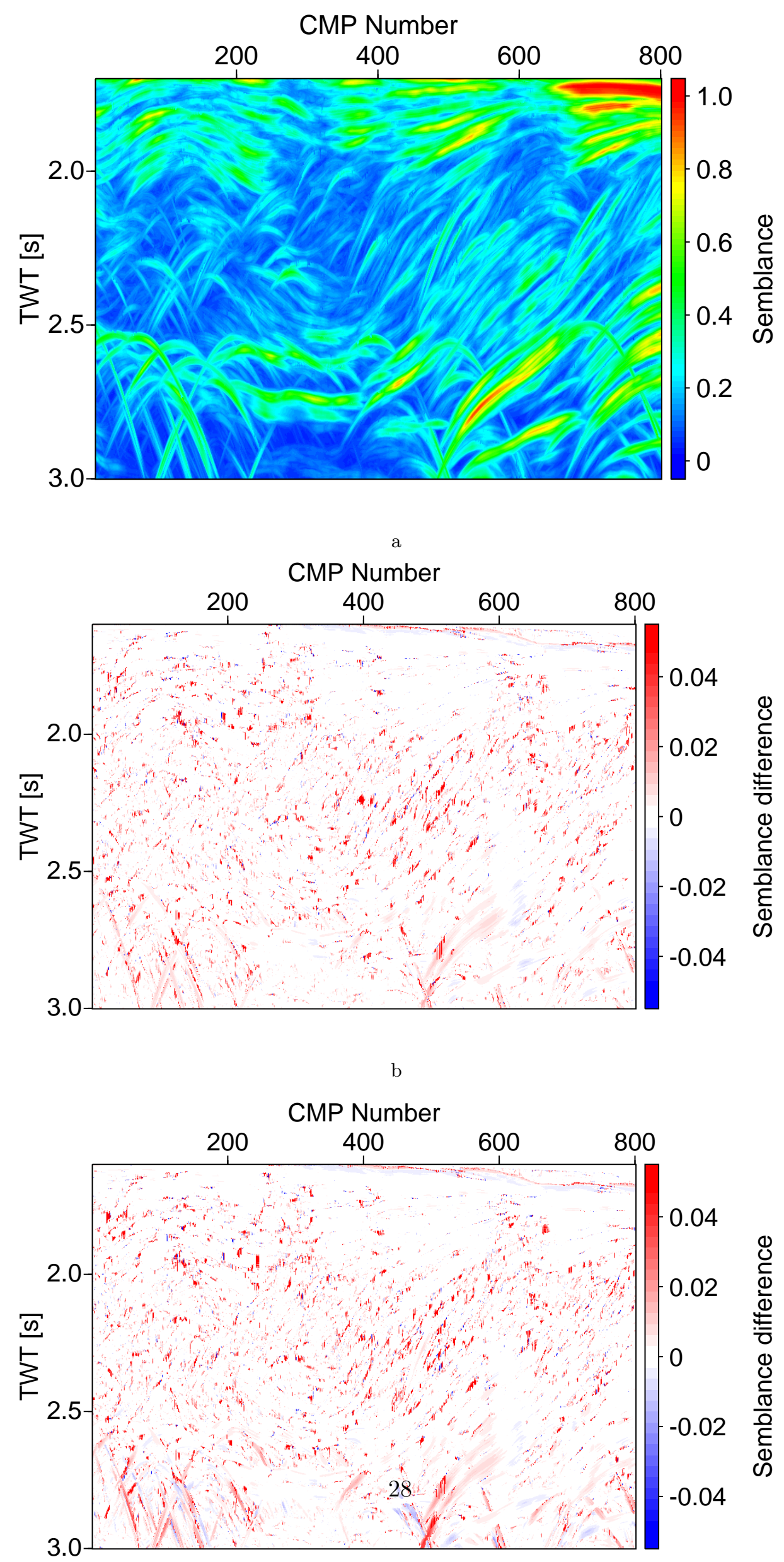

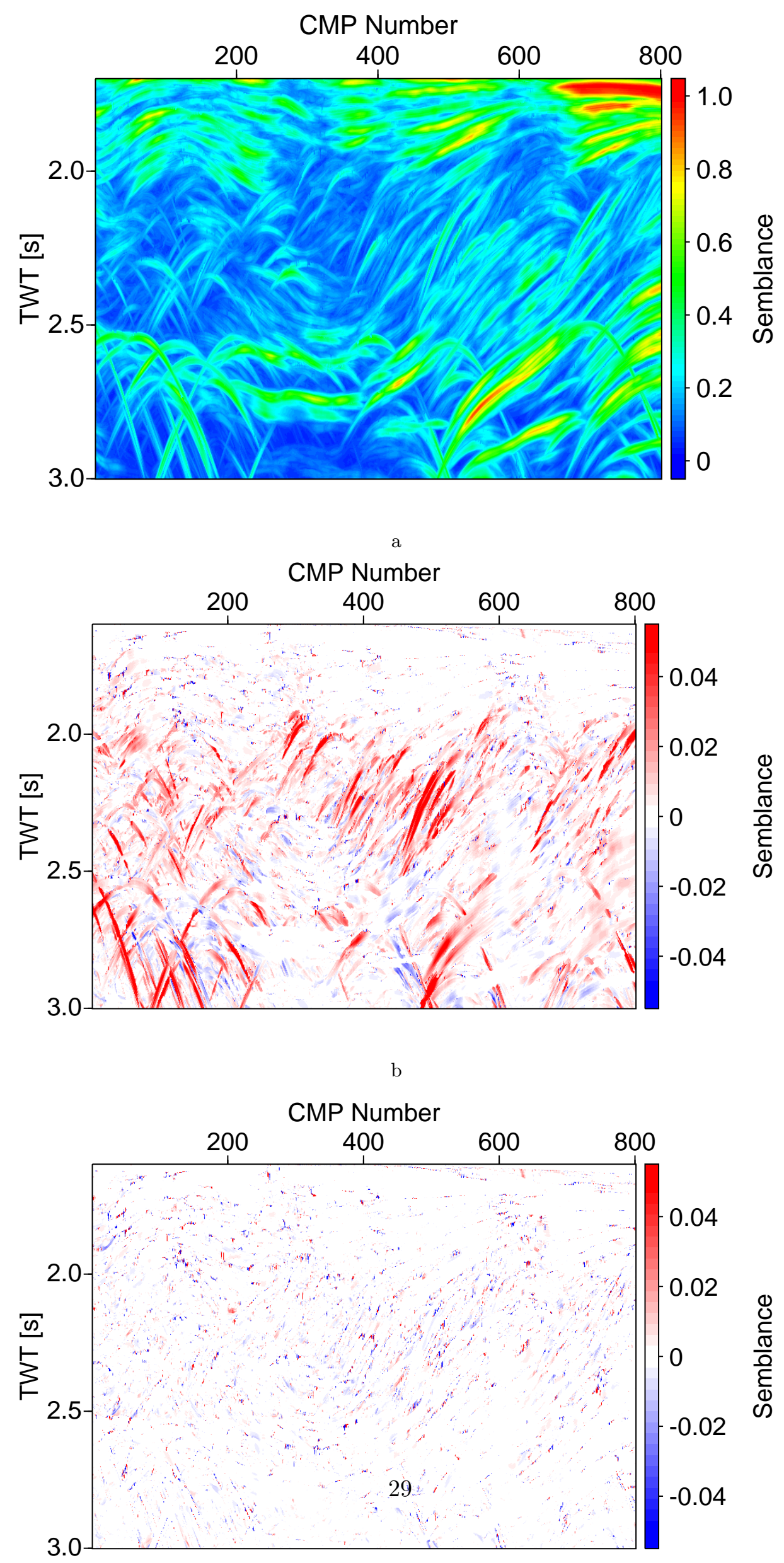

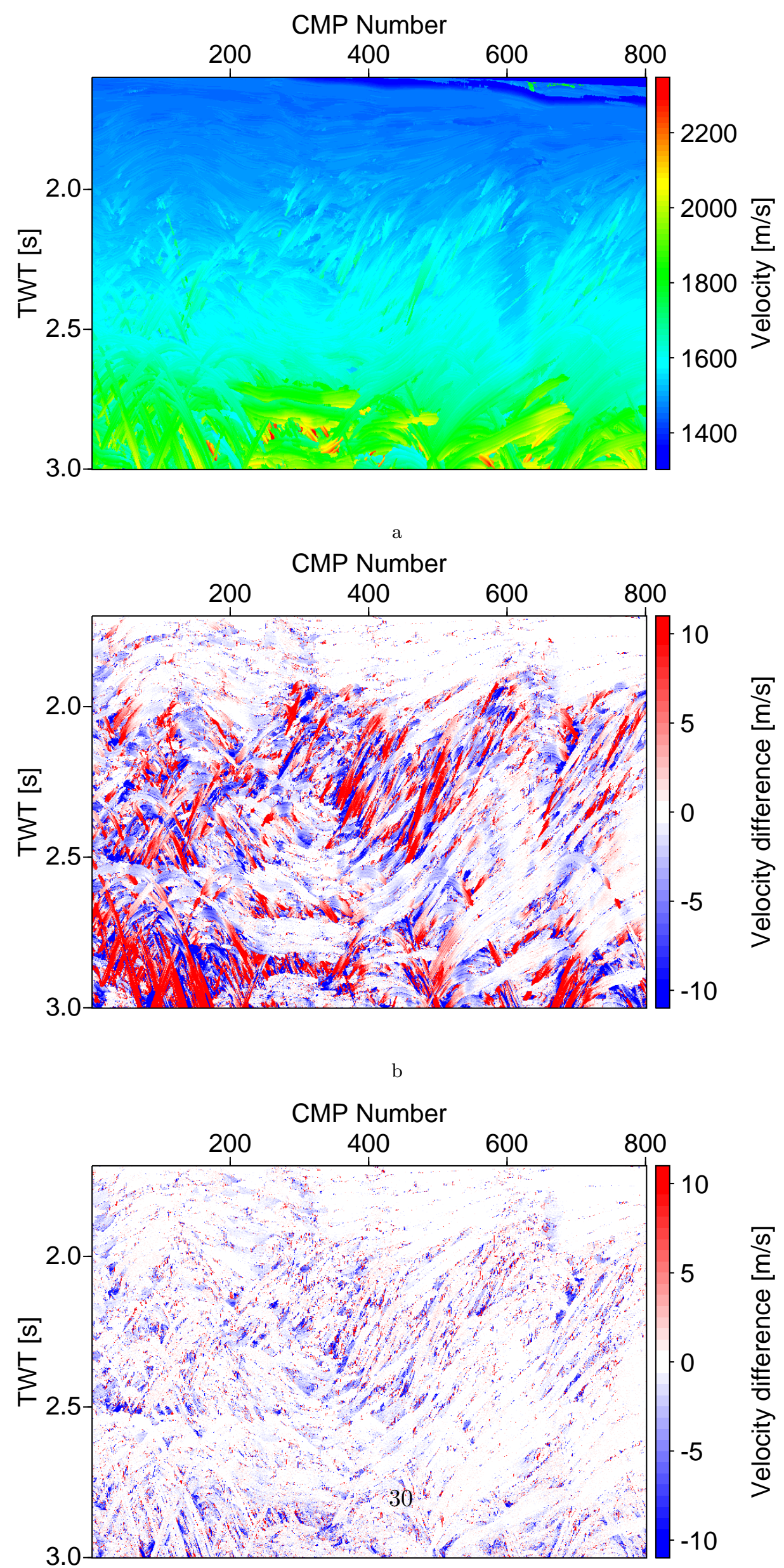


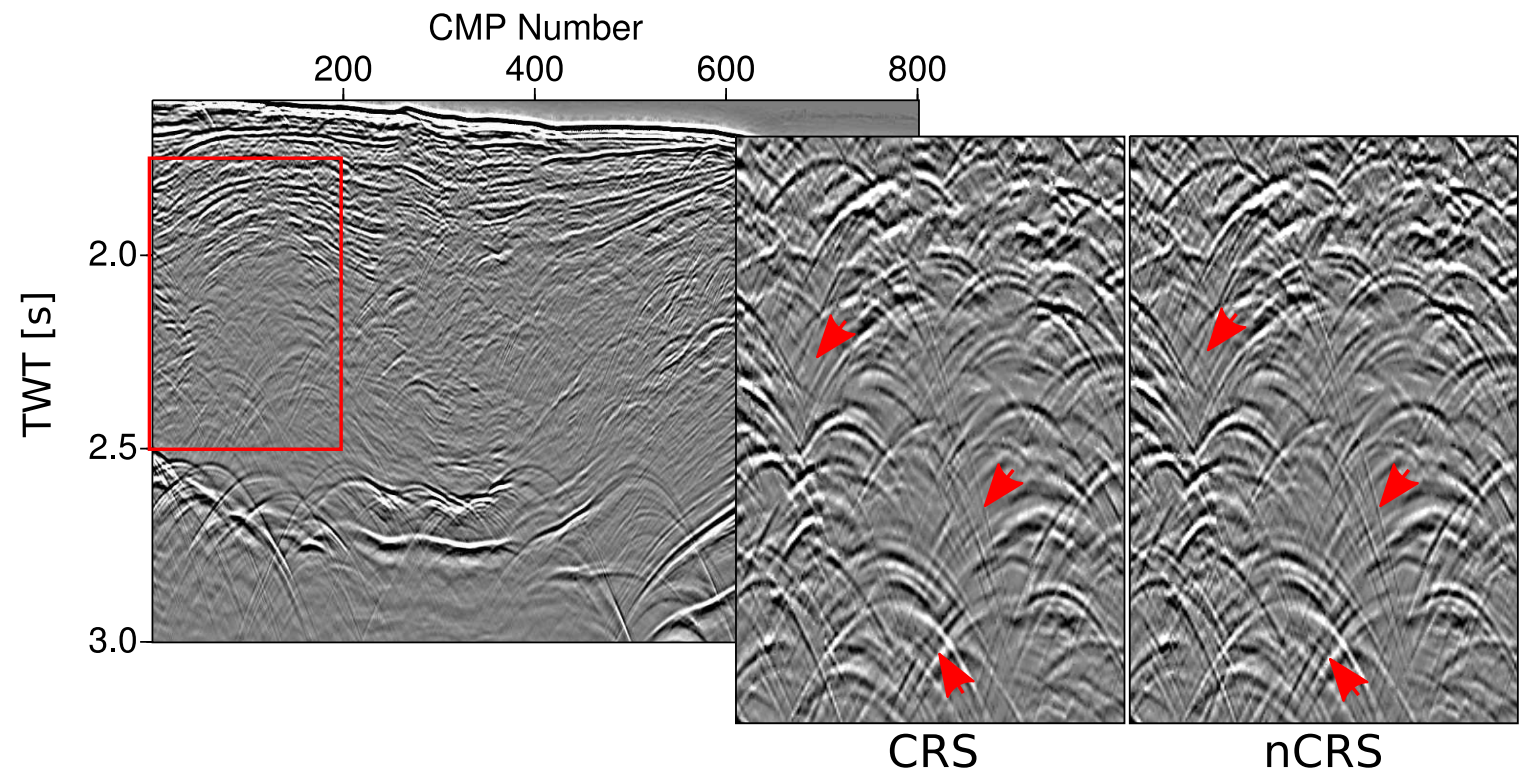

Figure 11: Stacked section of the fault system. The red box shows the excerpt shown on the right, where a diffraction separation is performed using attributes obtained by the CRS and nCRS operators. Red arrows indicate minor improvements achieved by nCRS compared to CRS. Most improvements concern the diffraction tails which are interesting for diffraction imaging. Multifocusing and iCRS are not shown since they performed similar to nCRS.

\section{Walda et al. -}




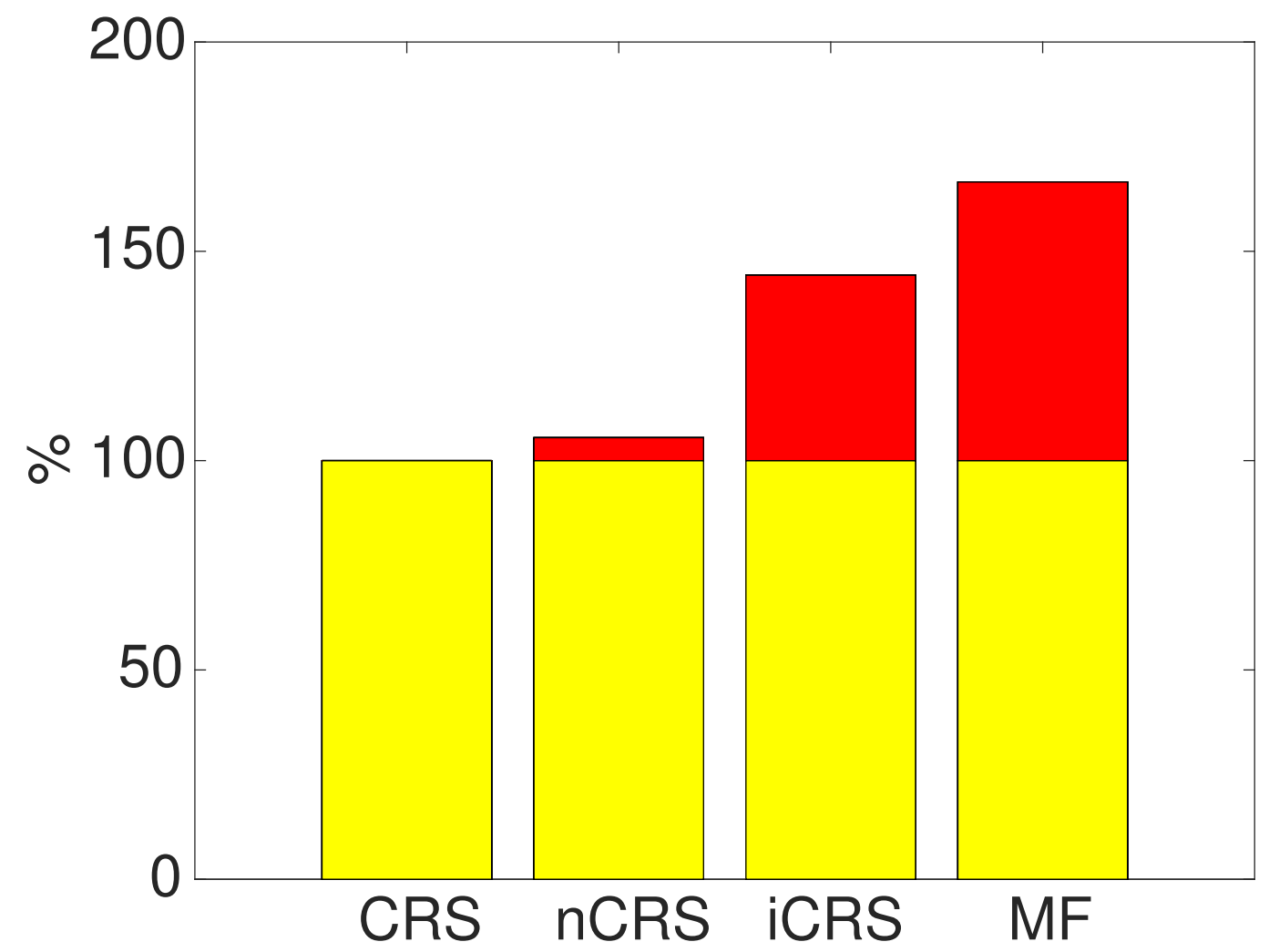

a

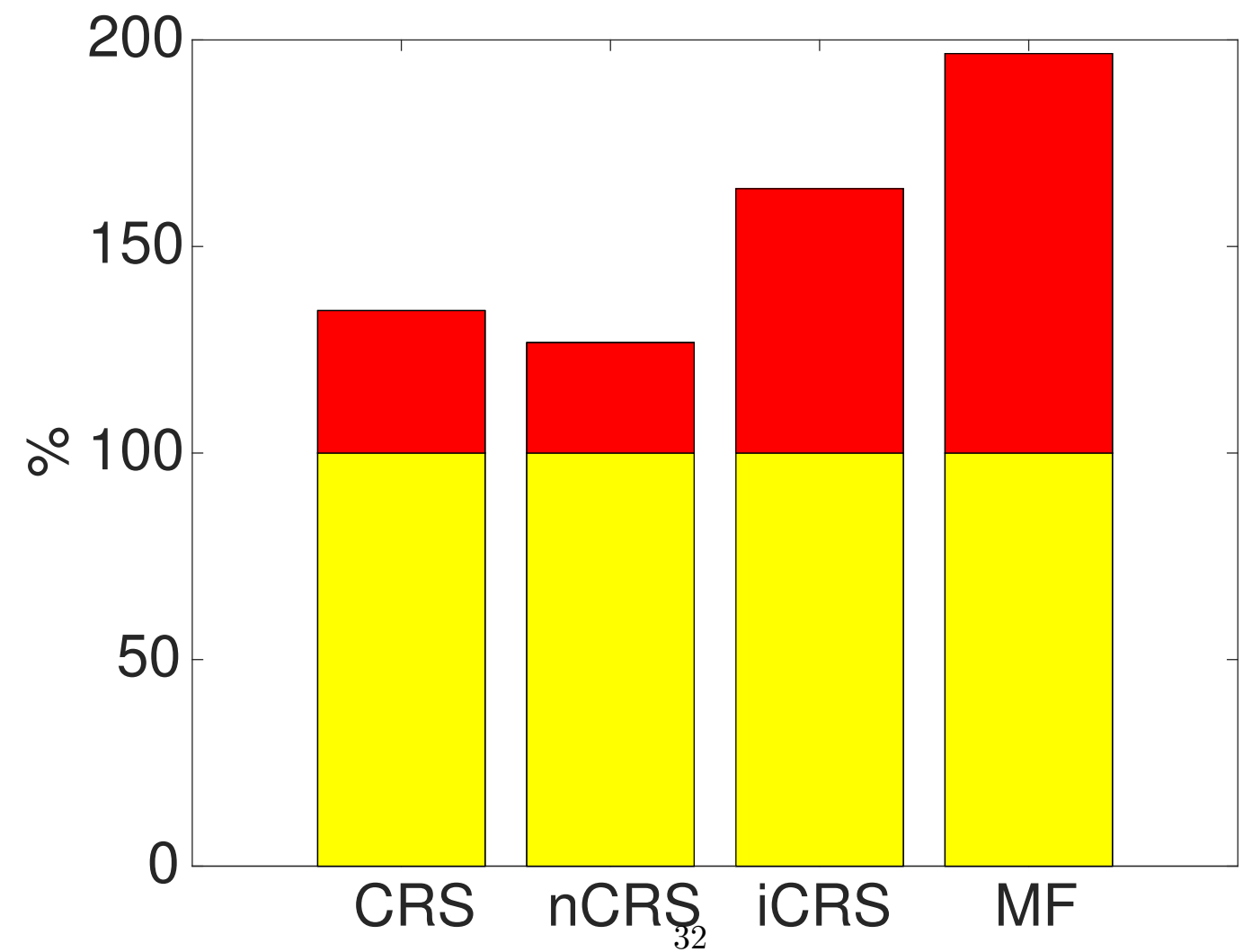

